\title{
Improving Tracking Performance of Predictive Functional Control Using Disturbance Observer and Its Application to Table Drive Systems
}

\author{
T. Satoh, K. Kaneko, N. Saito
}

\author{
Toshiyuki Satoh, Kotaro Kaneko, Naoki Saito \\ Akita Prefectural University \\ Department of Machine Intelligence and Systems Engineering \\ 84-4, Aza-Ebinokuchi, Tsuchiya, Yurihonjo, Akita, Japan \\ E-mail: tsatoh@akita-pu.ac.jp, m12a011@akita-pu.ac.jp \\ naoki_saito@akita-pu.ac.jp
}

\begin{abstract}
A practical approach for improving tracking performance of the predictive functional control (PFC) is proposed. The disturbance observer is utilized to nominalize the actual plant and to reduce the predicted output error in the PFC algorithm by canceling not only constant but also high-order disturbances. The proposed control scheme is experimentally validated on a single axis table drive system and is compared with the standard PFC and the industrial cascade control. The experimental results prove the effectiveness of the proposed disturbance observer-based PFC scheme.

Keywords: predictive functional control, disturbance observer, table drive system, model predictive control
\end{abstract}

\section{Introduction}

The model-based predictive control (MPC) has been successfully applied mainly in the petrochemical industry since it was put into practical use in the 1970's. In general, the quadratic optimization problem must be solved on-line to compute the optimal control sequence in the standard constrained multivariable MPC algorithm [14]. When the MPC is applied to the control of mechatronic servo systems, the timeconsuming optimization may be problematic since the sampling period of such systems is usually less than a few milliseconds and may be too short to complete the optimization.

On the other hand, a simple MPC scheme called the predictive functional control (PFC) [11-13] is widely used. Unlike the standard MPC, the PFC is primarily intended to single-input-single-output systems, and no on-line optimization is required since the control input is expressed as a linear combination of time-dependent basis functions. Although the PFC algorithm is much simpler than the standard MPC algorithm, it gives a similar performance to the full MPC. Accordingly, a variety of successful industrial applications have been reported (e.g., [2-5, 7, 8, 10,20]).

The tracking performance attained by the PFC heavily depends on the accuracy of the internal model of the actual plant. So, if the predicted output error caused by disturbances or model uncertainties is significant, the tracking performance may deteriorate, and the designed response cannot be obtained on the actual system. Another weakness of the PFC is that it cannot cope with high-order disturbances, such as ramp or parabola disturbances. Assume that disturbances entering the system are the $1 / s^{k}$-type $(k \geqslant 1)$. Then, for non-integrating plants, it is known that the PFC is offset-free for stepwise (i.e., $k=1)$ disturbances, which means that the stepwise disturbance is asymptotically rejected. However, this property does not hold true for high-order (i.e., $k \geqslant 2$ ) disturbances ${ }^{1}$. Also, for integrating plants, even stepwise disturbances cannot be rejected in the PFC scheme.

\footnotetext{
${ }^{1}$ Among high-order disturbances, ramp disturbances are especially worth considering. Examples of ramp disturbances include the viscous friction under uniform accelerated motion in mechatronic systems, or flow fluctuations in petrochemical plants.
} 
The purpose of this paper is to propose a practical way to overcome the above-mentioned drawbacks. To this end, we construct a dual loop control structure which consists of an inner loop formed by the disturbance observer (DOB) $[15,16,19]$ and an outer loop formed by the PFC. The DOB is known to be an effective compensation mechanism which reduces the influence of disturbances, uncertainties and nonlinearities in the plant and enforces the nominal input/output behavior on the actual plant especially in the low frequency range where the frequency of the reference signal concentrates. Accordingly, the actual plant behaves as if it is the nominal plant by introducing the DOB. In the DOB design, we can specify the number of integrators introduced in the loop transfer function. Hence, the DOB has the ability to asymptotically reject high-order disturbances as well as stepwise disturbances.

The contributions of the paper are as described below. First, it offers a way of improving the prediction accuracy within the PFC algorithm. This leads to the improvement of the transient performance of the PFC in the presence of disturbances and/or modeling errors since the DOB nominalizes the actual plant especially at low frequencies. Second, it solves the inherent weakness of the PFC against the highorder disturbances. When the $1 / s^{k}$-type $(k \geqslant 2)$ disturbances are applied, those disturbances cannot be rejected by the standard PFC controller. However, due to the introduction of the DOB, such high-order disturbances can be asymptotically rejected since the DOB has the specified number of integral action.

We should mention here that the idea of the combination of the PFC and the disturbance observer has originated in the recent application to the control of a pneumatic artificial muscle actuator by the authors [18]. However, in-depth argument about the advantages of the proposed scheme was not provided there. The present paper states the advantages that were left unclear in [18]. In addition, the proposed method is validated on a single axis table drive system and compared with the standard PFC and the industrial cascade control which consists of the inner proportional-plus-integral control loop and the outer proportional control loop. We also examine the performance differences between two different control systems that have the same PFC controller but different DOBs.

\section{Predictive Functional Control Enhanced with Disturbance Observer}

\subsection{Predictive Functional Control}

In this subsection, we give a brief overview of the predictive functional control (PFC). For more details of the PFC algorithm based on the state-space model, see, for example, S. Abu el Ata-Doss et al. [1].

Figure 1 shows the basic concept of the PFC. Suppose that the current time is labeled as time step $k$. A set-point trajectory is defined as a command signal which the process output $y_{P}$ should follow, and the value of the set-point trajectory at the current time step is denoted by $c(k)$. Also shown is a reference trajectory denoted by $y_{R}$. This trajectory starts at the current process output $y_{P}(k)$ and defines a desired trajectory along which the process output $y_{P}$ should approach the set-point trajectory. On the reference trajectory, there are a few coincidence points on which the performance index is defined so that the process output $y_{P}$ will coincide with the reference trajectory $y_{R}$. As an example, three coincidence points are drawn in Figure 1. The optimal control input trajectory is then computed on the basis of the predicted output. Once we have computed a future control input trajectory, we apply only the first element to the process. At the next time step, we repeat the whole cycle from the definition of the reference trajectory to the application of the first element of the optimal control input trajectory. We call this way of control a receding horizon control.

Next, we show the basic PFC algorithm which is a slightly modified formulation for handling time delay. If we set $d=0$ in the following description, the control law accords with the one without time delay. In the following, let $\mathbb{R}^{n}$ and $\mathbb{Z}^{n}$ be the set of all $n$-dimensional real vectors and the set of all $n$ dimensional integer vectors, respectively. Now assume that the plant is stable and has the time delay of $L$ and that the sampling period is $T_{s}$. The development of the PFC algorithm is based on the following 
SISO discrete-time linear state-space model of the plant:

$$
\left\{\begin{aligned}
x_{M}(k+1) & =A_{M} x_{M}(k)+B_{M} u(k), \\
y_{M}(k) & =C_{M} x_{M}(k)
\end{aligned}\right.
$$

where $x_{M} \in \mathbb{R}^{n}$ is the state vector, $u \in \mathbb{R}$ is the control input, $y_{M} \in \mathbb{R}$ is the model output, respectively. Here, the model output $y_{M}(k)$ is used to predict the future plant output $\hat{y}_{P}(k+d)$ where $d \in \mathbb{Z}$ is defined as the nearest integer of $L / T_{s}$. Assume that the following condition holds:

$$
\operatorname{det}\left(\begin{array}{cc}
A_{M}-I & B_{M} \\
C_{M} & 0
\end{array}\right) \neq 0
$$

Then the reference trajectory is defined as follows:

$$
y_{R}(k+d+i):=c(k+d+i)-\alpha^{i}\left(c(k+d)-\hat{y}_{P}(k+d)\right), i=0,1, \ldots
$$

where $\alpha \in \mathbb{R}$ is a parameter which adjusts the approaching ratio of the reference trajectory to the set-point $(0<\alpha<1)$. For example, Dieulot et al. [3] have chosen the parameter $\alpha$ as $\alpha=e^{-3 T_{s} / T_{\mathrm{CLTR}}}$ along with the following three coincidence points:

$$
\left(\begin{array}{lll}
h_{1} & h_{2} & h_{3}
\end{array}\right)=\left(\begin{array}{lll}
\frac{T_{\mathrm{CLTR}}}{3 T_{s}} & \frac{T_{\mathrm{CLTR}}}{2 T_{s}} & \frac{T_{\mathrm{CLTR}}}{T_{s}}
\end{array}\right)
$$

where $T_{\mathrm{CLTR}} \in \mathbb{R}$ is constant and called the desired closed-loop time response, which is taken as the time required to reach $95 \%$ of the final value [13]. In many cases, the performance index is defined as the quadratic sum of the errors between the predicted process output $\hat{y}_{P}$ and the reference trajectory $y_{R}$ as follows:

$$
J(k):=\sum_{j=1}^{n_{h}}\left\{\hat{y}_{P}\left(k+d+h_{j}\right)-y_{R}\left(k+d+h_{j}\right)\right\}^{2}
$$

where $h_{j} \in \mathbb{Z}(j=0,1, \ldots, h)$ and $n_{h} \in \mathbb{Z}$ are respectively the coincidence time point and the number of coincidence points. In the PFC, the future control input computed at each sampling instant is assumed to be the sum of weighted basis functions, and a time-dependent polynomial basis is usually employed. Then the optimal control input that minimizes the performance index (5) is given by

$$
u(k)=k_{0}\left\{c(k+d)-y_{P}(k)\right\}-\sum_{m=1}^{d_{e}} k_{m} e_{m}(k+d)+\tilde{v}_{x}^{T} x_{M}(k)+\tilde{v}_{x d}^{T} x_{M}(k-d)
$$

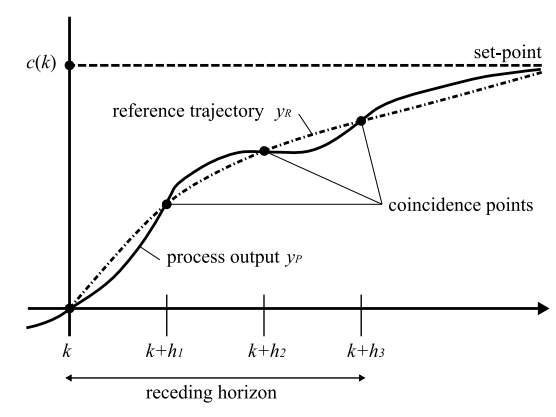

Figure 1: Concept of the predictive functional control. 
where $d_{e} \in \mathbb{Z}$ and $e_{m} \in \mathbb{Z}$ respectively denote the degree of the polynomial and unknown coefficients that are used when the predicted error at the future time step $k+d+i$ is approximated by a time-dependent polynomial, and $k_{0} \in \mathbb{R}, k_{m} \in \mathbb{R}, \tilde{v}_{x} \in \mathbb{R}^{n}$ and $\tilde{v}_{x d} \in \mathbb{R}^{n}$ are respectively given by

$$
k_{0}=v^{T}\left(\begin{array}{c}
1-\alpha^{h_{1}} \\
1-\alpha^{h_{2}} \\
\vdots \\
1-\alpha^{h_{n_{h}}}
\end{array}\right), \quad k_{m}=v^{T}\left(\begin{array}{c}
h_{1}^{m} \\
h_{2}^{m} \\
\vdots \\
h_{n_{h}}^{m}
\end{array}\right), \quad \tilde{v}_{x}=-\left(\begin{array}{c}
C_{M}\left(A_{M}^{h_{1}}-\alpha^{h_{1}} I\right) \\
C_{M}\left(A_{M}^{h_{2}}-\alpha^{h_{2}} I\right) \\
\vdots \\
C_{M}\left(A_{M}^{h_{h_{h}}}-\alpha^{h_{n_{h}} I}\right)
\end{array}\right)^{T} v, \quad \tilde{v}_{x d}=-\left(\begin{array}{c}
\left(\alpha^{h_{1}}-1\right) C_{M} \\
\left(\alpha^{h_{2}}-1\right) C_{M} \\
\vdots \\
\left(\alpha^{h_{h_{h}}}-1\right) C_{M}
\end{array}\right)^{T} v
$$

In (7), $v \in \mathbb{R}^{h_{n_{h}}}$ is given by

$$
v=\left(\begin{array}{lll}
y_{B}\left(h_{1}\right) & \cdots & y_{B}\left(h_{n_{h}}\right)
\end{array}\right)^{T}\left(\sum_{j=1}^{n_{h}} y_{B}\left(h_{j}\right) y_{B}\left(h_{j}\right)^{T}\right)^{-1} U_{B}(0)
$$

where $y_{B}\left(h_{j}\right)=\left(\begin{array}{lll}y_{B_{1}}\left(h_{j}\right) & \cdots & y_{B_{n_{B}}}\left(h_{j}\right)\end{array}\right)^{T} \in \mathbb{R}^{h_{n_{h}} \times n_{B}}$ and $U_{B}(0)=\left(\begin{array}{llll}1 & 0 & \cdots & 0\end{array}\right)^{T} \in \mathbb{Z}^{n_{B}}$. Here, $y_{B_{l}}(i) \in \mathbb{R}$ is the forced response to the basis function of the form $i^{l-1}\left(l=1,2, \ldots, n_{B}\right)$.

The second term in the right-hand side of (6) denotes the term to compensate for the prediction error due to disturbances and/or uncertainties. The unknown coefficients $e_{m}(k+d)\left(m=1,2, \ldots, d_{e}\right)$ are determined in the following fashion. First, the number of steps $h_{c}$ used for the polynomial approximation is specified. Then, at each sampling instant, the following vectors $\varphi \in \mathbb{R}^{h_{c}}, \theta \in \mathbb{R}^{d_{e}}$ and a matrix $H \in \mathbb{Z}^{h_{c} \times d_{e}}$ are defined:

$\varphi:=\left(\begin{array}{c}e(k+d)-e\left(k+d-h_{c}\right) \\ e(k+d-1)-e\left(k+d-h_{c}\right) \\ \vdots \\ e\left(k+d-h_{c}+1\right)-e\left(k+d-h_{c}\right)\end{array}\right), \quad H:=\left(\begin{array}{cccc}h_{c} & h_{c}^{2} & \ldots & h_{c}^{d_{e}} \\ h_{c}-1 & \left(h_{c}-1\right)^{2} & \ldots & \left(h_{c}-1\right)^{d_{e}} \\ \vdots & \vdots & \ddots & \vdots \\ 1 & 1 & \ldots & 1\end{array}\right), \quad \theta:=\left(\begin{array}{c}e_{1}(k+d) \\ e_{2}(k+d) \\ \vdots \\ e_{d_{e}}(k+d)\end{array}\right)$.

The problem is to approximate $\varphi$ by $H \theta$ in the least-squares sense. The vector of unknown coefficients $\theta$ that minimizes the square error $J(\theta):=(\varphi-H \theta)^{T}(\varphi-H \theta)$ is hence given by

$$
\theta=\left(H^{T} H\right)^{-1} H^{T} \varphi
$$

Once the number of steps $h_{c}$ is specified, the matrix $\left(H^{T} H\right)^{-1} H^{T}$ can be preliminarily computed off-line. Therefore, the optimal coefficients $e_{m}(k)\left(m=1,2, \ldots, d_{e}\right)$ can be determined by just updating the vector $\varphi$ and computing (10) on-line. The mechanism which extrapolates the past prediction error to the future prediction horizon by using the coefficients given in (10) is called the auto-compensation. The use of the auto-compensation is optional, so the PFC is often utilized without the second term in the right-hand side of (6).

\subsection{Disturbance Observer}

The disturbance observer (DOB) has the structure depicted in Figure 2 where $P(s)$ is the transfer function of the real plant, $P_{n}(s)$ is the nominal model of the plant, and $Q(s)$ is a proper and stable filter. The nominal model $P_{n}(s)$ is supposed to be minimum-phase in the following. On the assumption that disturbances acting on the system, modeling errors and nonlinearities can be regarded as an equivalent 


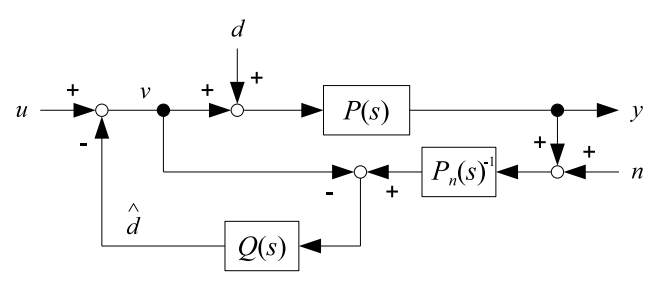

Figure 2: Structure of the disturbance observer. Figure 3: Equivalent form of the disturbance observer.

disturbance $d$ at the plant input, the DOB computes the estimate $\hat{d}$ of the current disturbance $d$, which is subtracted from the input $u$ to cancel the disturbance.

The designed parameter of the DOB is the filter $Q(s)$. This filter is closely related to the sensitivity properties of the DOB. For example, the transfer function from the disturbance $d$ to the measurement output $y$ is given by

$$
G_{y d}(s)=\frac{(1-Q(s)) P(s) P_{n}(s)}{P_{n}(s)+\left(P(s)-P_{n}(s)\right) Q(s)} .
$$

Accordingly, to reject the disturbance $d$ asymptotically, the gain of $Q(s)$ should be unity at low frequencies. On the other hand, the transfer function from the measurement noise $n$ to the measurement output $y$ is given by

$$
G_{y n}(s)=\frac{-P(s) Q(s)}{P_{n}(s)+\left(P(s)-P_{n}(s)\right) Q(s)} .
$$

Hence, to suppress the measurement noise, the gain of $Q(s)$ should be 0 at high frequencies. Therefore, $Q(s)$ should be a low-pass filter with the DC gain of 1 . Since the closed-loop transfer function from the input $u$ to the measurement output $y$ in Figure 2 is given by

$$
G_{y u}(s)=\frac{P(s) P_{n}(s)}{(1-Q(s)) P_{n}(s)+P(s) Q(s)},
$$

the transfer property approximates the nominal plant $P_{n}(s)$ at low frequencies if $Q(s)$ is chosen as stated above. This means that the DOB can nominalize the real plant at low frequencies.

When the plant has no unstable zeros, the low-pass filter $Q(s)$ can be selected as [19]

$$
Q(s)=\frac{1+\sum_{m=1}^{n_{q}-\rho_{q}} f_{m} s^{m}}{1+\sum_{m=1}^{n_{q}} f_{m} s^{m}}
$$

where $n_{q} \in \mathbb{Z}, \rho_{q} \in \mathbb{Z}$ and $f_{m} \in \mathbb{R}$ are respectively the order of $Q(s)$, the relative degree of $Q(s)$ and unknown coefficients to be determined. To make $Q(s) P_{n}(s)^{-1}$ proper, $\rho_{q}$ must be chosen as $\rho_{q} \geqslant \rho_{P}$ where $\rho_{P} \in \mathbb{Z}$ is the relative degree of $P_{n}(s)$. Figure 3 shows the equivalent form of the disturbance observer. In the figure, $(1-Q(s))^{-1}$ can be written as

$$
\frac{1}{1-Q(s)}=\frac{1+\sum_{m=1}^{n_{q}} f_{m} s^{m}}{s^{n_{I}}\left(f_{n_{I}}+\sum_{m=1}^{\rho_{q}} f_{n_{I}+m} s^{m}\right)}
$$

where $n_{I}=n_{q}-\rho_{q}+1$, if the $Q$-filter of the form given in (14) is used. Hence, we can see that the loop transfer function includes $n_{I}$ integrators in Figure 3, which means that the DOB can asymptotically reject up to the $1 / s^{n_{I}}$-type disturbances.

Various types of analogue filters can be utilized to realize $Q(s)$, and in many cases, the Butterworth or the binomial low-pass filters are used. More specifically, the Butterworth filter is a reasonable option 

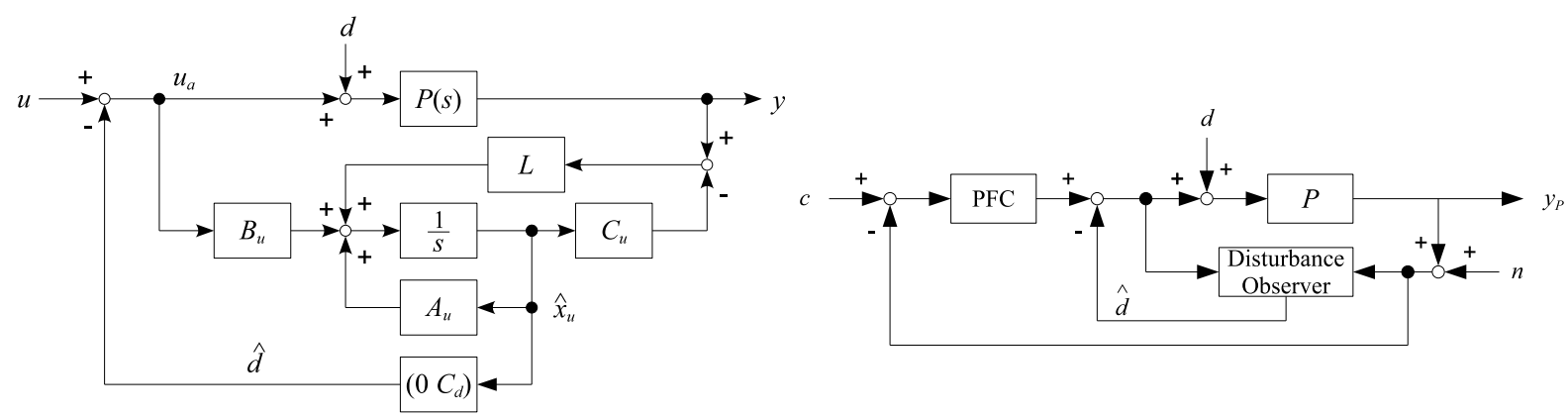

Figure 5: Structure of the proposed control system.

Figure 4: Structure of the unknown input distur-

bance observer.

when $n_{q}=\rho_{q}$, which means that the degree of the numerator polynomial of $Q(s)$ is 0 . Otherwise, the binomial filter of the following form is useful:

$$
Q(s)=\frac{1+\sum_{m=1}^{n_{q}-\rho_{q}} a_{m}\left(s \tau_{n_{q}}\right)^{m}}{1+\sum_{m=1}^{n_{q}} a_{m}\left(s \tau_{n_{q}}\right)^{m}}
$$

where $a_{m}\left(m=1,2, \ldots, n_{q}\right)$ are the binomial coefficients $\left(i . e ., a_{m}=n_{q} ! / m !\left(n_{q}-m\right) !\right)$ and $\tau_{n_{q}}$ is a design variable to be determined.

Aside from the DOB, another type of disturbance observer based on the unknown input observer, which is referred to as the unknown input disturbance observer (UIDOB), has also been proposed [6]. The UIDOB is a natural extension of the Luenberger observer, and it is based on the following state-space models of the plant and the fictitious disturbance generator:

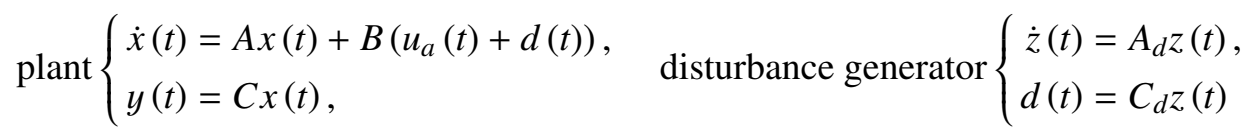

where $x \in \mathbb{R}^{n}$ is the state variable of the plant, $u_{a} \in \mathbb{R}$ is the control input, $y \in \mathbb{R}$ is the plant output, $z \in \mathbb{R}^{n_{d}}$ is the state variable of the disturbance generator and $d \in \mathbb{R}$ is the disturbance. Notice that the eigenvalues of $A_{d}$ are not restricted to those on the origin (i.e., disturbances are not necessarily the $1 / s^{k}$ type). It is assumed that the pair $(C, A)$ and $\left(C_{d}, A_{d}\right)$ are both observable and that no eigenvalues of $A_{d}$ coincide with zeros of the plant. The UIDOB is based on the augmented system obtained from (17) and is given by

$$
\left\{\begin{array}{l}
\left(\begin{array}{l}
\dot{\hat{x}}(t) \\
\dot{\hat{z}}(t)
\end{array}\right)=\left(\begin{array}{cc}
A & B C_{d} \\
0 & A_{d}
\end{array}\right)\left(\begin{array}{l}
\hat{x}(t) \\
\hat{z}(t)
\end{array}\right)+\left(\begin{array}{l}
B \\
0
\end{array}\right) u_{a}(t)+L\left\{y(t)-\left(\begin{array}{ll}
C & 0
\end{array}\right)\left(\begin{array}{l}
\hat{x}(t) \\
\hat{z}(t)
\end{array}\right)\right\}, \\
\left(\begin{array}{l}
\hat{x}(t) \\
\hat{d}(t)
\end{array}\right)=\left(\begin{array}{cc}
I & 0 \\
0 & C_{d}
\end{array}\right)\left(\begin{array}{l}
\hat{x}(t) \\
\hat{z}(t)
\end{array}\right)
\end{array}\right.
$$

where $\hat{x}, \hat{z}$ and $\hat{d}$ are the estimates of $x, z$ and $d$, respectively. $L \in \mathbb{R}^{n+n_{d}}$ is the observer gain which is chosen to stabilize the observer system. Figure 4 shows the structure of the UIDOB where

$$
A_{u}=\left(\begin{array}{cc}
A & B C_{d} \\
0 & A_{d}
\end{array}\right), B_{u}=\left(\begin{array}{l}
B \\
0
\end{array}\right), C_{u}=\left(\begin{array}{ll}
C & 0
\end{array}\right), \hat{x}_{u}(t)=\left(\begin{array}{l}
\hat{x}(t) \\
\hat{z}(t)
\end{array}\right) .
$$

The estimate $\hat{d}$ is fed back to the control input to compensate for the input disturbance $d$. Unlike the DOB, the UIDOB estimates $\hat{x}$ as well as $\hat{d}$, so the observer-based state feedback technique can be applicable to stabilize the plant. 
The equivalence and difference between the DOB and UIDOB has been already investigated by Schrijver and van Dijk [9] $]^{2}$, and they have shown that the DOB is equivalent to the UIDOB for some specific design choices. Actually, the DOB is more general than the UIDOB for the following reasons [9]:

- Given any UIDOB designed for the $1 / s^{k}$-type disturbance model, we can always find exactly the same DOB as the given UIDOB.

- It is possible to design a DOB by freely specifying the relative degree of the $Q(s)$. However, in the UIDOB case, the relative degree always becomes $\rho_{P}+1$ where $\rho_{P}$ is the relative degree of the plant. Hence, it is generally impossible to convert a given DOB into an equivalent UIDOB structure.

- There is no freedom to choose the order of $Q(s)$ in the UIDOB structure, and the order of the UIDOB is higher than that of the DOB for plants with stable zeros.

We therefore use the DOB in this paper. Refer to Schrijver and van Dijk [9] for more details about the DOB and UIDOB.

\subsection{Structure of Proposed Control System}

The structure of the overall control system we propose is shown in Figure 5 where $P$ represents the actual plant, $c$ is the set-point, $y_{P}$ is the plant output, $d$ is the disturbance, $n$ is the noise, and $\hat{d}$ is the estimated disturbance. The control system consists of an inner loop formed by a disturbance observer and an outer loop formed by a PFC controller.

The advantages of using the disturbance observer along with the predictive functional control are twofold.

First, an improvement of prediction accuracy in the PFC algorithm is expected since the input/output behavior of the actual plant approaches its nominal characteristics by using the DOB. This can be understood in the following way. As explained in the previous subsection, the design parameter $Q(s)$ is a low-pass filter, and its DC gain is usually chosen to be $1(=0 \mathrm{~dB})$ to reject the $1 / s^{k}$-type disturbances asymptotically. Hence, in the low-frequency band on which disturbances generally concentrate, the transfer function from the control input to the plant output given in (13) can be approximated by

$$
G_{y u}(s)=\frac{P(s) P_{n}(s)}{(1-Q(s)) P_{n}(s)+P(s) Q(s)} \simeq P_{n}(s)
$$

since $Q(s) \simeq 1$. This means that the behavior of the actual plant equipped with the DOB shown in Figure 2 is close to that of the nominal model $P_{n}(s)$ as long as the bandwidth of $Q(s)$ is properly designed. Although gain mismatches or modeling errors between the internal model and the real plant are unavoidable in real-world applications, the PFC has tolerance for such uncertainties to some extent. However, the transient performance may deteriorate even though the steady-state response is satisfactory. For instance, suppose that the real plant $P(s)$ and the internal model $P_{n}(s)$ are both stable first-order systems and given by as follows:

$$
P(s)=\frac{k}{s+p}, \quad P_{n}(s)=\frac{k_{n}}{s+p}
$$

where $k>0, k_{n}>0$ and $p>0$. If there exists a gain mismatch between these two transfer functions such that $k<k_{n}$, then the closed-loop response is slower than the designed response based on the internal model $P_{n}(s)$. This difference is due to the inaccuracy of the prediction in the PFC algorithm. The use of

\footnotetext{
${ }^{2}$ The DOB is denoted as the disturbance estimation filter (DEF) in [9].
} 
Table 1: Specifications of linear actuator (left) and DC motor (right).

\begin{tabular}{cc|cc}
\hline Description & Value & Description & Value \\
\hline \hline ball screw lead & $10 \mathrm{~mm}$ & nominal voltage & $24 \mathrm{~V}$ \\
stroke & $500 \mathrm{~mm}$ & rated current & $2.96 \mathrm{~A}$ \\
rail length & $670 \mathrm{~mm}$ & rated torque & $0.28 \mathrm{Nm}$ \\
maximum rotational speed & $500 \mathrm{~mm} / \mathrm{s}$ & rated speed & $1810 \mathrm{r} / \mathrm{min}$ \\
\hline
\end{tabular}

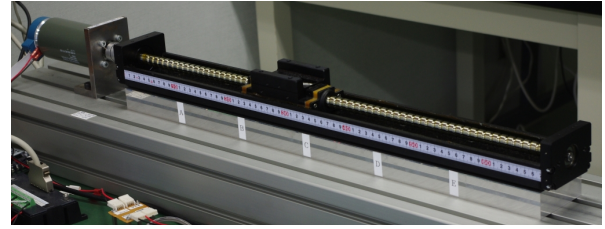

Figure 6: Single axis table drive system.

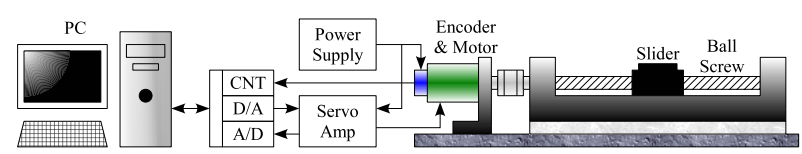

Figure 7: Schematic diagram of experimental setup.

the disturbance observer improves the prediction accuracy and, in consequence, leads to the improvement of the transient response in the PFC scheme.

Second, the disturbance observer can compensate for high-order (e.g., ramp, parabola, etc.) disturbances. It is known that, in the case of non-integrating processes, the steady-state error caused by stepwise disturbances becomes zero in the PFC scheme. Unfortunately, this property does not hold true for high-order disturbances. Also, for integrating processes, the steady-state error remains even though the disturbance is stepwise. To eliminate the steady-state error in such cases, one or more integrators must be added in the loop transfer function. This can be accomplished within the local loop formed by the disturbance observer when $n_{I} \geqslant 1$ in (15), and the disturbance is asymptotically eliminated in the inner-loop shown in Figure 5. Consequently, the disturbance rejection property of the PFC can be enhanced, and the above-mentioned difficulty is remedied by the simultaneous use of the disturbance observer.

\section{Controller Design for Single Axis Table Drive System}

\subsection{Plant Description}

Figure 6 shows the external view of the single axis table drive system which is used in the experiment shown in Section 4. Also, Figure 7 shows the schematic diagram of the experimental setup. As is clear from Figure 7, the system is controlled by the semi-closed-loop control method. The core of this experimental apparatus is a single axis linear actuator (NSK Ltd., Monocarrier MCM08050H10K), which is driven by an 80 Watt DC servo motor (maxon motor ag, F2260.885) equipped with an optical encoder with the resolution of 1000 pulses/revolution. The DC motor is driven by a DC servo amplifier (maxon motor ag, ADS 50/5). The specifications of the linear actuator and DC motor are summarized in Table 1. The encoder pulse is received by a 24-bit encoder counter board (Interface Co., Ltd., PCI-6204) and counted by quad edge evaluation. The command signal to the DC servo amplifier is sent from a 12bit digital-to-analogue converter, and the current monitor signal from the DC servo amplifier is received by a 12-bit analog-to-digital converter. Those $\mathrm{D} / \mathrm{A}$ and $\mathrm{A} / \mathrm{D}$ converters are both implemented on one analogue I/O board (Interface Co., Ltd., PCI-3521). The control PC is equipped with dual $1.7 \mathrm{GHz}$ Intel Xeon ${ }^{\circledR}$ processors and is running on Microsoft Windows $2000^{\circledR}$ operating system. A real-time control environment is constructed by using MATLAB ${ }^{\circledR}$, Simulink ${ }^{\circledR}$ and Real-Time Windows Target ${ }^{\circledR}$ (Math- 
Works, Inc.), and the table drive system is controlled at the sample rate of $1 \mathrm{kHz}$. Two S-function blocks for the use of PCI-6204 (encoder counter board) and PCI-3521 (A/D and D/A board) were developed by the authors since Simulink is not shipped with blocks for these boards.

We use the DC servo amplifier in the current control mode to command and control the motor torque instead of directly manipulating the motor current. Hence, on the assumption that the table drive system can be approximated by a one-inertia system, its equation of motion subject to friction torque is given by

$$
\ddot{\theta}(t)=\frac{K_{S}}{J} e_{a}(t)-\frac{1}{J} T_{d}(t)
$$

where $\theta$ denotes the angle of the motor shaft, $e_{a}$ is the command voltage to the DC servo amplifier, $J$ is the equivalent moment of inertia, $K_{S}$ is the conversion factor from the command voltage to the motor torque, and $T_{d}$ is the nonlinear friction torque. Notice that $T_{d}$ includes the viscous friction torque. In the case of our experimental apparatus, the conversion factor is given by $K_{S}=0.0801 \mathrm{Nm} / \mathrm{V}$, and the nominal value of the moment of inertia is identified as $J=1.6928 \times 10^{-4} \mathrm{~kg} \mathrm{~m}^{2}$ by using a standard system identification technique.

We define the state vector and output variables as $\left(x_{1} x_{2}\right)^{T}:=(\theta \dot{\theta})^{T}$ and $y:=\theta$, respectively. Also, the time delay of the experimental apparatus can be negligible as long as the sampling period is greater than or equal to $1 \mathrm{~ms}$. Then the state-space description of the single axis table drive system is given by

$$
\left\{\begin{aligned}
\left(\begin{array}{l}
\dot{x}_{1}(t) \\
\dot{x}_{2}(t)
\end{array}\right) & =\left(\begin{array}{ll}
0 & 1 \\
0 & 0
\end{array}\right)\left(\begin{array}{l}
x_{1}(t) \\
x_{2}(t)
\end{array}\right)+\left(\begin{array}{c}
0 \\
K_{S} / J
\end{array}\right) e_{a}(t)+\left(\begin{array}{c}
0 \\
-1 / J
\end{array}\right) T_{d}(t) \\
y(t) & =\left(\begin{array}{ll}
1 & 0
\end{array}\right)\left(\begin{array}{l}
x_{1}(t) \\
x_{2}(t)
\end{array}\right)
\end{aligned}\right.
$$

Here, the nonlinear friction characteristics of this single axis table drive system can be approximated by, for example, the general kinetic (GK) friction model [17] of the form

$$
T_{d}(t)= \begin{cases}\left\{T_{c}+\left(T_{b}-T_{c}\right) e^{-\left|\frac{\dot{\theta}(t)}{\dot{\theta}_{s t r}}\right|^{2}}\right\} \operatorname{sgn} \dot{\theta}(t)+D \dot{\theta}(t), & \text { if } \dot{\theta} \neq 0, \\ T_{e}(t), & \text { if } \dot{\theta}=0 \text { and }\left|T_{e}\right|<T_{s}, \\ T_{b} \operatorname{sgn} T_{e}(t), & \text { if } \dot{\theta}=0 \text { and }\left|T_{e}\right|>T_{s}\end{cases}
$$

where $\dot{\theta}_{s t r}$ is the Stribeck velocity, $T_{c}$ is the Coulomb friction level, $T_{b}$ is the breakaway torque, $D$ is the viscous friction coefficient, $T_{e}$ is the external torque generated by the driving motor. However, to evaluate the effectiveness of the disturbance observer, and to compare the prediction accuracy in the PFC algorithm with or without the disturbance observer, we treat the friction torque as unknown disturbance in this paper.

\subsection{Design of Disturbance Observer}

The nominal transfer function from the applied voltage to the angular velocity can be described as

$$
P_{n}(s)=\frac{473.2}{s}
$$

Hence, the relative degree of the $Q$-filter must be chosen as $\rho_{q} \geqslant 1$, and we take $\rho_{q}$ to be 1 in this paper.

The required number of the integral action introduced by the DOB depends on the nature of disturbances, and it is difficult to decide its appropriate number beforehand. So, we will design two DOBs to see the difference due to the number of integral action under the following design conditions: 


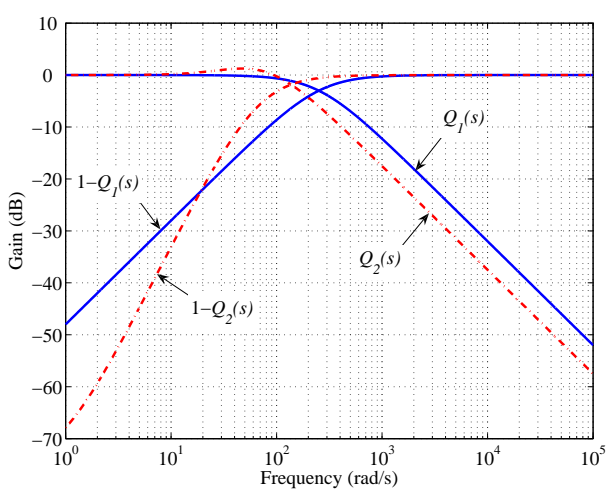

Figure 8: Frequency characteristics of $Q_{i}(s)$ and $1-Q_{i}(s)(i=1,2)$.

1. relative degree $\rho_{q}=1$, number of integral action $n_{I}=1$.

2. relative degree $\rho_{q}=1$, number of integral action $n_{I}=2$.

Since the transfer function from the input voltage $e_{a}$ to the rotor angle $\theta$ is given by the double integrator model $P_{n}(s) / s=473.2 / s^{2}$, the closed-loop system equipped with the DOB can asymptotically reject the stepwise disturbance when $n_{I}=2$. The order of the $Q$-filter $n_{q}$ can be determined by the relation $n_{q}=n_{I}+\rho_{q}-1$, so $n_{q}=1$ for the former case, and $n_{q}=2$ for the latter case.

First, we design a first order filter $Q_{1}(s)$ as an analogue Butterworth low-pass filter. The target cut-off frequency $\omega_{c}$ is chosen to be $40 \mathrm{~Hz}$. Then, $Q_{1}(s)$ is given by

$$
Q_{1}(s)=\frac{251.33}{s+251.33} .
$$

Next, we design a second order filter $Q_{2}(s)$. In this case, we use the prototype of the binomial filter given in (16). For the case of $Q_{2}(s)$, the binomial coefficients $a_{1}$ and $a_{2}$ are given as $a_{1}=2$ and $a_{2}=1$, respectively. To determine the parameter $\tau_{n_{q}}$, we numerically solve the following optimization problem:

$$
\min _{\tau_{n_{q}}} \sum_{i=1}^{N}\left(\left|Q_{d}\left(\omega_{i}\right)\right|-\left|Q_{2}\left(j \omega_{i}, \tau_{n_{q}}\right)\right|\right)^{2}
$$

where $\left|Q_{d}(\omega)\right|$ is the gain of the ideal low-pass filter whose value is 1 over the frequency range from 0 to $\omega_{c}$ and is 0 at higher frequencies than $\omega_{c}$, and $\omega_{i}(i=1,2, \ldots, N)$ are $N$ logarithmically-spaced points between decades $10^{-1} \mathrm{rad} / \mathrm{s}$ and $10^{4} \mathrm{rad} / \mathrm{s}$. Solving the optimization problem with $N=3000$, we obtain the optimal value of the parameter $\tau_{n_{q}}=0.015$, which results in the following second order filter:

$$
Q_{2}(s)=\frac{133.38(s+33.35)}{(s+66.69)^{2}} .
$$

Figure 8 shows the gains of $Q_{i}(s)$ and $1-Q_{i}(s)(i=1,2)$. We can see form Figure 8 that the property of the disturbance suppression of the DOB using $Q_{2}(s)$ is better than that using $Q_{1}(s)$.

\subsection{PFC Parameters}

Using the control structure depicted in Figure 5, we implement the PFC algorithm in the position control loop with the double integral internal model $P_{n}(s) / s$. Also, the time delay steps $d$ is fixed to 0 in the control law given in (6). 
To cope with non-constant references, both the step and ramp basis functions are employed which will be expected to achieve better tracking performance than the step basis function alone. We choose the desired closed-loop time response $T_{\text {CLTR }}$ to be $0.02 \mathrm{~s}$ to make a compromise between the stability of the closed-loop system and the tracking performance, and the sampling period $T_{s}$ of the system is fixed to $0.001 \mathrm{~s}$. The same approaching ratio and the coincidence points as Dieulot et al. [3] have used, which are already shown in Subsection 2.1, are employed. So, the approaching ratio becomes $\alpha=0.8607$, and the three coincidence points given in (4) are defined as $\left(h_{1} h_{2} h_{3}\right)=\left(\begin{array}{lll}6 & 10 & 20\end{array}\right)$.

In the experiment shown in the next section, we will also use the standard PFC control scheme with the auto-compensation for comparison, and the predicted error is approximated by a first degree polynomial. The number of steps used for the polynomial approximation in (9) is taken to be $h_{c}=20$. It can be seen that the tracking performance is improved as $h_{c}$ becomes small. However, in exchange for the improvement, the control effort becomes large and shows extremely oscillatory behavior. The number of steps 20 is therefore the result of compromise between the tracking performance and control effort.

\subsection{Design of Industrial Controller}

As well as the PFC plus auto-compensation scheme, we will use an industrial controller for comparison in the experiment. It consists of a proportional-integral controller in the inner speed loop and a proportional controller in the outer position loop. The current loop is neglected since the current is already controlled in the motor driver. The PI controller in the speed loop is defined as

$$
C_{P I}(s)=K_{P I}\left(1+\frac{D}{J s}\right)
$$

where $K_{P I}$ is the proportional gain. This PI controller is constructed to cancel the plant dynamics and force the open-loop transfer function to be $K_{S} K_{P I} / J_{S}$. It follows that the viscous friction is theoretically canceled. Here, the proportional gain $K_{P I}$ is chosen to be 1 . As the value of $K_{P I}$ is increased, the speed of response is improved, but the maximum tracking error is not. Also, the stability of the inner loop is sensitive to the change of this gain and easily collapses when $K_{P I}>1$. Hence, $K_{P I}$ is fixed to 1 .

The $\mathrm{P}$ controller in the position loop is defined as $C_{P}(s)=K_{P}$. On the simulation model, we can increase the proportional gain up to 100 . However, due to uncertainties in the actual plant and the nonlinear friction, the responses of the closed-loop system becomes highly oscillatory, and the large control effort is applied to the system when $K_{P}$ is greater than 20. Hence, $K_{P}$ is fixed to 20 in the experiment.

\section{Experimental Results}

To test the proposed control scheme, an experiment was conducted using a sinusoidal reference input. The amplitude and the angular frequency are respectively $5 \mathrm{rad}(7.96 \mathrm{~mm}$ in displacement) and $\pi / 4 \mathrm{rad} / \mathrm{s}$.

Figure 9 shows the reference and the motor angles obtained by different kinds of control schemes. In this and the subsequent figures, the correspondence between the legends and control schemes are as follows: 'PFC' is the standard PFC, 'PFC+AC' is the PFC with the auto-compensation, 'PFC+DOB1' is the PFC combined with the disturbance observer using $Q_{1}(s)$, 'PFC+DOB2' is the PFC combined with the disturbance observer using $Q_{2}(s)$, 'P+PI' is the industrial control. We can see from the top graph that only the standard PFC can hardly actuate the table drive system and cannot track the reference angle. This is mainly because the stiction torque (breakaway torque) is larger than the driving torque generated by the motor and, thus, the table drive system does not move. Every control scheme except the standard PFC tracks the reference, and it is hard to distinguish from each other in the top graph. So, a detailed 

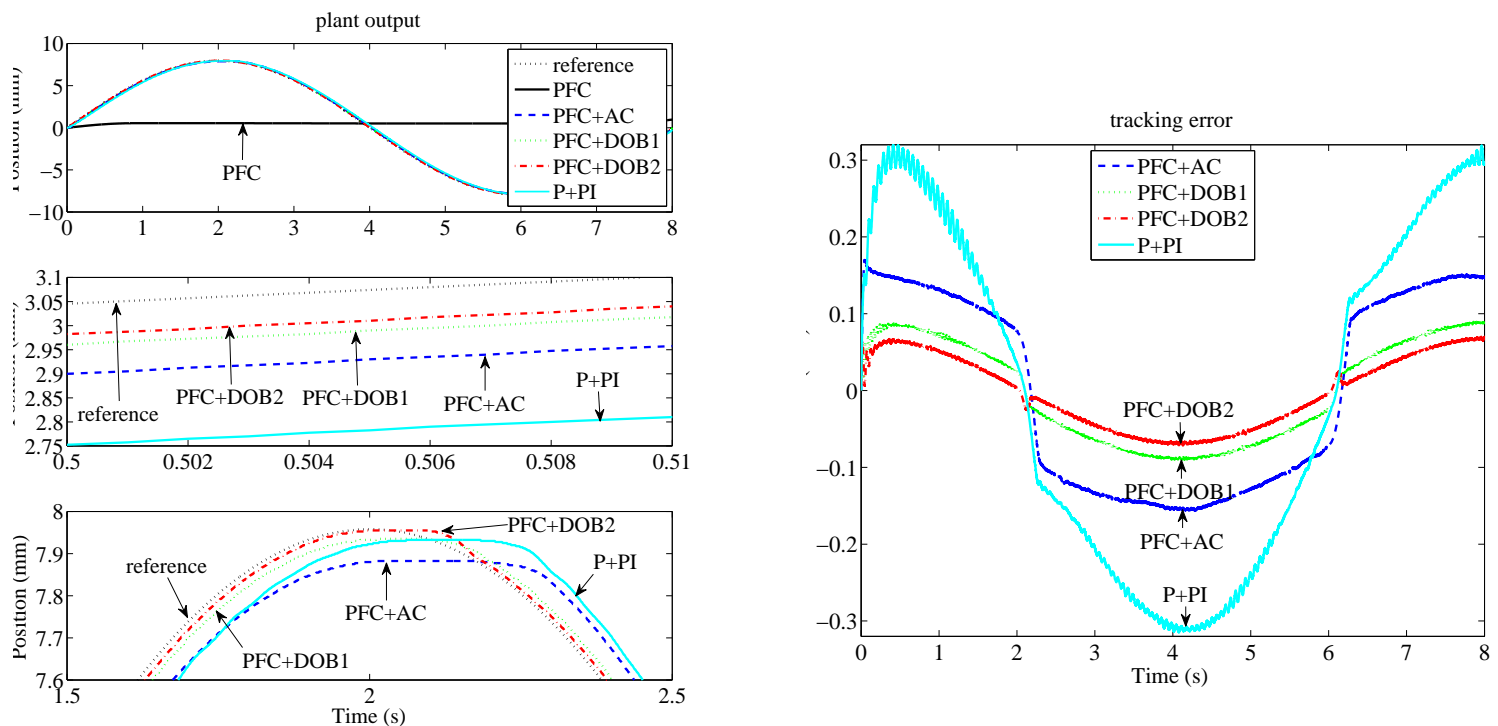

Figure 10: Comparison of tracking errors.

Figure 9: Comparison of control performances (top graph) and its closeups (middle and bottom graphs).

view near 0.5 seconds where the Coulomb friction torque and the viscous friction torque respectively act as the stepwise disturbance and the ramp disturbance is shown in the middle graph of Figure 9. In addition, a detailed view around 2 seconds where a change of direction of the movement occurs and therefore the friction torque takes the maximum is shown in the bottom graph of Figure 9. It can be seen from Figure 9 that the PFCs along with the disturbance observer track the reference better than other control schemes without the disturbance observer.

Figure 10 shows the tracking error of each control scheme where the result of the PFC is excluded from the graph since its tracking error is significant. Comparing the two PFC plus disturbance observer schemes, we see that the tracking performance of PFC+DOB2 is better than that of PFC+DOB1. The difference arises from the number of integrators in the velocity loop, and, as a result, the disturbance observer using $Q_{2}(s)$, which adds two integrators in the loop, has lower sensitivity and better disturbance rejection property at low frequencies. We also see that $\mathrm{PFC}+\mathrm{AC}$ is a simple but effective control scheme, considering the fact that it uses no active disturbance estimation or cancellation. However, the peak-to-peak value of its tracking error is about twice as large as that of PFC+DOB1. Also, since the auto-compensation is based on the past prediction errors and extrapolation, a certain amount of degradation of the tracking error is unavoidable when the reference signal changes its direction; see around 2 seconds in Figure 10. Finally, we can see that the industrial controller $(\mathrm{P}+\mathrm{PI})$ provides the worst tracking performance.

Figure 11 shows the control effort of each control scheme. Basically, the magnitude is similar to each other. Every control effort presents oscillatory behavior during the phase of initiation of motion (see from 0 to 2 seconds), though that of the industrial controller especially draws our attention. It can be seen from the bottom graph that, with the disturbance observer, the control effort quickly responds to the change of direction of the reference to cancel the disturbance torque. This leads to the improvement of the tracking error on the time interval between $2.1 \mathrm{~s}$ and $2.2 \mathrm{~s}$.

Figure 12 shows the actual plant output and the predicted output of the inner model. Here, to compare the prediction accuracy inside the pure PFC algorithm, the PFC scheme using the auto-compensation $(\mathrm{PFC}+\mathrm{AC})$ is excluded from the comparison. The top graph is in the case of the PFC without the distur- 

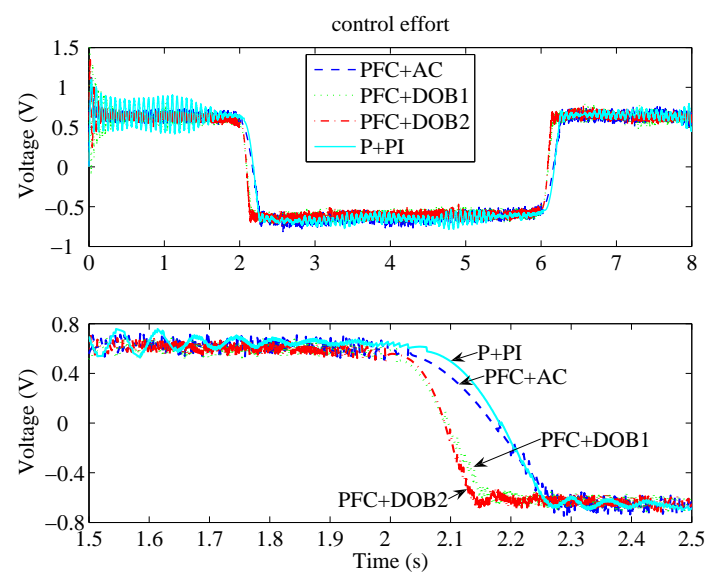

Figure 11: Comparison of control inputs (top graph) and its closeup (bottom graph).
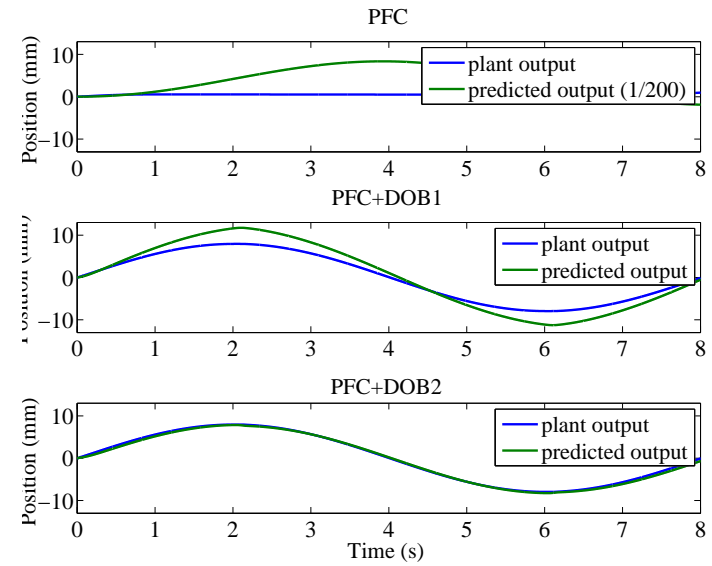

Figure 12: Comparison of prediction accuracy.

Table 2: Root Integrated Squared Values.

\begin{tabular}{c||c|c}
\hline Control Scheme & Tracking Error & Control Effort \\
\hline \hline PFC & 11.45 & 1.78 \\
PFC+DOB1 & 0.14 & 2.12 \\
PFC+DOB2 & 0.10 & 2.13 \\
P+PI & 0.48 & 2.23 \\
PFC+AC & 0.32 & 2.19 \\
\hline
\end{tabular}

bance observer. Since the amplitude of the predicted output is too large in this case, two-hundredth of its value is drawn in the figure. As we have already seen in Figure 9, the ordinary PFC cannot overcome the friction torque, and the table drive system does not start to move. Hence, the predicted output is completely different from the plant output. On the other hand, we can see from the middle and bottom figures that the predicted output approximates the actual plant output. Also, the disturbance observer using $Q_{2}(s)$ provides a more accurate result than the one using $Q_{1}(s)$. In the middle figure, the magnitude of the predicted output is larger than the plant output, which, roughly speaking, indicates that the gain of the nominal model is larger than the actual plant. It follows that the speed of response of the plant becomes slower than the designed response. This can be confirmed by comparing the response of the PFC using $Q_{1}(s)$ with that of the PFC using $Q_{2}(s)$ in Figure 9. Even though the specified desired closed-loop time response $T_{\text {CLTR }}$ is the same in both cases, the former slightly lags behind the latter, which is very close to the designed response owing to the accurate prediction.

To see the difference of the control performances quantitatively, we show the root integrated squared values of the tracking error $e$ and that of the control effort $u$ computed on the time interval between $0 \mathrm{~s}$ and $8 \mathrm{~s}$ in Table 2. Clearly, the PFC plus the disturbance observer using $Q_{2}(s)$ provides the best performance of all the control schemes. On the other hand, in terms of the control effort, we can hardly recognize a difference apart from the standard PFC. As a consequence, we can see that, in this experiment, the PFC combined with the disturbance observer successfully improved the tracking performance without putting extra control energy into the system. 


\section{Conclusions}

We have presented a disturbance observer-based approach for improving the tracking performance of the predictive functional control. The role of the disturbance observer is to reject unknown disturbances including the plant-model mismatch or nonlinearities that can be regarded as an input disturbance and to nominalize the real plant. Owing to the disturbance observer, the predicted output error in the PFC algorithm is reduced, and, as a result of this, the tracking performance is improved. The proposed control scheme was implemented and validated on a single axis table drive system. It was confirmed that the predicted output error was effectively reduced and that better tracking performance was provided than the standard PFC using the auto-compensation mechanism and the conventional P plus PI control scheme even in the severe environment where the system was not driven by the standard the PFC scheme due to friction. Future works involves the extension to non-minimum phase linear systems.

\section{Acknowledgment}

This work was supported by the Japan Society for the Promotion of Science under Grant-in-Aid for Scientific Research (C) 21560247.

\section{Bibliography}

[1] S. Abu el Ata-Doss, P. Fiani and J. Richalet, "Handling input and state constraints in predictive functional control," Proc. of the 30th Conference on Decision and Control, pp. 985-990, Brighton, England, 1991.

[2] N. Bigdeli and M. Haeri, Predictive functional control for active queue management in congested TCP/IP networks, ISA Transactions, vol. 48, no. 1, pp. 107-121, 2009.

[3] J.Y. Dieulot, T. Benhammi, F. Colas, P.J. Barre, Composite predictive functional control strategies, application to positioning axes, International Journal of Computers, Communications $\mathcal{E}$ Control, vol. III, no. 1, pp. 41-50, 2008.

[4] D. Dovžan and I. Škjrank, Predictive functional control based on an adaptive fuzzy model of a hybrid semi-batch reactor, Control Engineering Practice, vol. 18, no. 8, pp.979-989, 2010.

[5] M. Hadjiski and V. Asenov, Predictive functional control using a blending approach, Cybernetics and Information Technologies (Bulgarian Academy of Science), vol. 5, no. 2, pp. 32-41, 2005.

[6] C. Johnson, "Accommodation of external disturbances in linear regulator and servomechanism problems," IEEE Transactions on Automatic Control, vol. 16, no. 6, pp. 635-644, 1971.

[7] M. Lepetič, I. Škrjanc, H. G. Chiacchiarini and D. Matko, Predictive functional control based on fuzzy model: comparison with linear predictive functional control and PID control, Journal of Intelligent and Robotic Systems, vol. 36, pp. 467-480, 2003.

[8] M. Primucci and M. Basualdo, Thermodynamic predictive functional control applied to CSTR with jacket system, Proc. of IFAC 15th Triennial World Congress, Barcelona, Spain, 2002.

[9] E. Schrijver and J. van Dijk, "Disturbance observers for rigid mechanical systems: equivalence, stability, and desigh," ASME Journal of Dynamic Systems, Measurement, and Control, vol. 124, pp. 539-548, 2002. 
[10] I. Škrjanc and D. Matko, Predictive functional control based on fuzzy model for heat-exchanger pilot plant, IEEE Transactions on Fuzzy Systems, vol. 8, no. 6, pp. 705-712, 2000.

[11] J. Richalet, S. Abu el Ata-Doss, C. Arber, H.B. Kuntze, A. Jacubasch and W. Schill, Predictive functional control: application to fast and accurate robot, Proc. of IFAC 10th World Congress, Munich, Germany, 1987.

[12] J. Richalet, Industrial applications of model based predictive control, Automatica, vol. 29, no. 5, pp. 1251-1274, 1993.

[13] J. Richalet and D. O'donovan, Predictive Functional Control: Principles and Industrial Applications. London, England: Springer-Verlag, 2009.

[14] J. M. Maciejowski, Predictive Control with Constraints. Harlow, England: Pearson Education, 2002.

[15] K. Ohishi, M. Nakao, K. Ohnishi and K. Miyachi, Microprocessor-controlled DC motor for loadinsensitive position servosystem, IEEE Transactions on Industrial Electronics, vol. 34, no. 1, pp.4449, 1987.

[16] T. Murakami and K. Ohnishi, Advanced motion control in mechatronics - A tutorial, Proc. of the IEEE International Workshop on Intelligent Control, Istanbul, Turkey, vol. 1, pp. SL9-SL17, 1990.

[17] E. G. Papadopoulos and G. C. Chasparis, Analysis and model-based control of servomechanisms with friction, ASME Journal of Dynamic Systems, Measurement, and Control, pp. 911-915, 2004.

[18] T. Satoh, N. Saito and N. Saga, Predictive functional control with disturbance observer for pneumatic artificial muscle actuator, Proc. of the 1st International Conference on Applied Bionics and Biomechanics, Venice, Italy, no page number, 2010.

[19] T. Umeno and Y. Hori, Robust speed control of DC servomotors using modern two-degrees-offreedom controller design, IEEE Trans. on Industrial Electronics, vol. 38, no. 5, pp. 363-368, 1991.

[20] A. Vivas and P. Poignet, Model based predictive control of a fully parallel robot, Control Engineering Practice, vol. 13, no. 7, pp. 863-874, 2005. 